\title{
Assessing the benefit of treatment in chronic inflammatory demyelinating polyneuropathy: the challenges of clinical practice
}

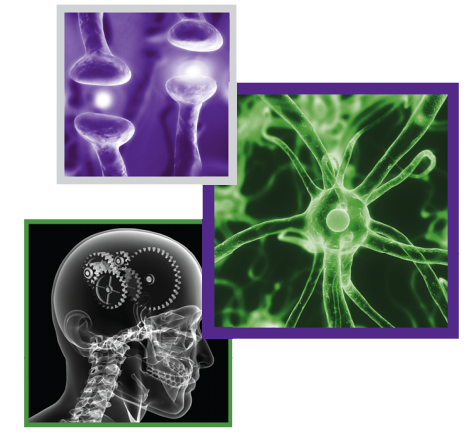

\author{
Yusuf A Rajabally*,1,2 \\ ${ }^{1}$ Aston Brain Centre, School of Life \& Health Sciences, Aston University, Birmingham, UK \\ ${ }^{2}$ Regional Neuromuscular Clinic, University Hospitals Birmingham, Birmingham, UK \\ *Author for correspondence: y.rajabally@aston.ac.uk
}

First draft submitted: 3 July 2018; Accepted for publication: 4 July 2018; Published online: 18 September 2018

Keywords: benefit $\bullet$ CIDP $\bullet$ outcome $\bullet$ treatment

Chronic inflammatory demyelinating polyneuropathy (CIDP) is an immune-mediated motor and/or sensory disorder of the peripheral nerves which presents in various forms, resulting in functional neurological deficits. The prevalence of CIDP is low, of around 5 per 100,000 [1] but it is a disorder which responds to treatment, among which corticosteroids, intravenous immunoglobulins (IVIg) and plasma exchanges. The issue around evaluating effects of the treatment of CIDP is of great importance, both in avoiding deterimental indesirable effects as well as ensuring optimum cost-to-benefit ratio.

As a result, effective, reliable and reproducible ways of assessing the benefit of treatment or the lack of it, are essential in clinical practice. In its typical form, CIDP presents with proximal and distal weakness of the four limbs. Sensory loss to large fibre modalities predominate. Motor deficits frequently represent the basis of disability. The evaluation of treatment effects has for many clinicians, been reliant on Medical Research Council (MRC) scores of groups of muscles, pre- and post-therapy. However, in sensory predominant forms strength scores are unhelpful. Use of disability measures are often not systematic and individualized goals, if used, may not infrequently be based on imprecise subjective descriptions, sometimes in retrospect. IVIg has not been demonstrated as effective on improving strength scores in meta-analyses of clinical trials performed, whereas they have been shown to improve disability scores. Different scales were however used in different trials and their clinical equivalence is not established. Transformation of various scales in a modified Rankin Scale equivalent allowed to demonstrate benefit from three pooled studies of 84 participants in the last Cochrane review [2].

Disability scales are often considered by clinicians mainly as research tools and their implementation in day-today practice is variable. There are many reasons for this, including lack of time for detailed assessments, lack of familiarity with scales, as well as focus on traditional methods of evaluation which often include priority given to subjective patient descriptions. Also, the simple lack of interest by treating physicians, is a non-negligible factor. In recent years, emphasis has been put on the use of meaningful assessment tools, albeit essentially in the research arena, with increasing evidence of the inappropriateness of traditional MRC scores in the first instance. A new Rasch-built MRC grading has been proposed [3] but its clinical usage and uptake, has not been evaluated. The Inflammatory Neuropathy Cause and Treatment Group (INCAT) scale, consisting of an upper and a lower limb disability level component, represents a method used by many in practice [4]. A newer version, the Overall Neuropathy Limitation Scale (ONLS), consisting of a slight variation of the leg score, has, similarly, been adopted by others [5]. Despite poor content and structural validity, these scales are considered reliable and responsive [6]. The minimum detectable change with the scale was however found greater than the minimal clinically important difference (MCID) which suggests suboptimal sensitivity to detect improvement in some subjects, compounded by low emphasis on proximal upper limb function and stamina-related tasks [6]. In more recent years, a Rasch-built Overall Disability Scale (RODS) for Guillain-Barré syndrome, CIDP and gammopathy-related polyneuropathy has been developed, with construction of a 24-item scale consisting of tasks of increasing difficulty, with a conversion into a centile metric 
score, varying from 0 (greatest activity and social participation limitation) to 100 (no activity and social participation limitation) [7]. Compared with the ONLS, this 'Inflammatory RODS' (I-RODS) offered assessment of a wider range of item difficulties and therefore evaluation of patients of different ability. Still more recently, grip strength, using a Martin vigorimeter or Jamar dynamometer has been described as a reliable measure of treatment effect in CIDP $[8]$ and correlations have been found with the ONLS and I-RODS $[9,10]$.

The practical usage of disability scales in patients with CIDP is more often than not straight forward. Scales such as the INCAT and ONLS offer rapid evaluation of change, with minimal examiner training and take a very short time to administer. A clinician familiar with the scale is usually able to score an individual patients in a few minutes. Similarly, the I-RODS scale, although lengthier, can, with adequate directives to patients, rapidly and easily be completed by themselves prior to or at attendance or be evaluated similarly within a few minutes by the clinician. Grip strength measurements also represent a very convenient and and quick evaluation method, which in view of recent studies, may in certain instances even supersede above-mentioned scales.

Difficulties in the use of these assessment tools are however real for the clinician. The INCAT and ONLS may not detect meaningful change in an improved patient and may not ascertain important deficits preventing repetitive movements or tasks. The I-RODS may not be culturally adapted to all subjects and may entail uncetainties and confusion for individual patients, for example, in separating what, for some individual items, is 'difficult' or 'not difficult'. Furthermore, the impact of the repetitive nature of such full, all-item assessments on responses provided has not been studied, particularly in patients who deem themselves stable, although this may result in bias. The use of these scales is also complicated by associated conditions and for example, concurrent rheumatological, cardiovascular or respiratory disease and even potentially more so, depressive illness, can make scores very challenging for adequate interpretation. Delineating what is purely related to the neuropathy, often as a result, requires in clinical practice, more than these scores. Individual patients' level of functioning may otherwise also vary considerably. In the very active subject, the 'ability to run' or 'walk for a kilometre' may not reflect the actual limitation experienced and it is not uncommon to hear descriptions of disabling deficits preventing more demanding tasks which they previously performed without problem. Similarly, in patients severely disabled at baseline, recovering upper limb ability allowing to write, turn pages of a book, use a spoon to feed or use a mobile phone, tablet or remote control, can be of paramount importance to quality of life. These are however not precisely considered in the items of existing scales for CIDP. Disability scores, in clinical practice may not always fully replace for those reasons, the need for detailed history-taking and adequate questioning regarding most important functions for the individual at baseline, in comparison with premorbid state as well as with their current global impairment. Strength score testing as well as sensory, particularly propioceptive, examination remain similarly important to separate neurological deficits from those resulting from other causes. Further evaluations with 10-m timed walks and 6-min walking distance, may all represent potentially useful measures in selected cases. Tailoring the assessment to the needs of the patient is therefore essential in the task of monitoring patients with CIDP.

Another important question when scales are used in CIDP is how much improvement is genuinely clinically meaningful? The answer to this remains also central to the issue of clinical monitoring as has major implications on subsequent therapeutic decisions. Traditionally, an improvement of MRC scores by a total of two points in at least two muscles was often considered as a marker of objective improvement. The recent study on the poor reliability of the MRC scale [3] casts significant doubt on such a definition. The use of disability scales themselves require establishing clinically meaningful cut-offs for change, to define improvement or deterioration. The concept of MCID appears relevant and fundamental in clinical practice as much as research, although is commonly overlooked. The complexity surrounding the MCID, which also depends on patients' perspectives of the relevance of individual scale items as well on scale dynamics in relation to disease subtype (particularly with the heterogeneity of CIDP), makes it difficult in practice to agree on what score change is meaningful [11]. This in turn leads to situations in clinical practice which are difficult to interpret and still more difficult to act upon. An change of one point on the ONLS/INCAT has been found to correspond to one of about four points on the I-RODS [12]. A score improvement from three to two for the arm component of the ONLS, with the patient describing being able to do buttons and zips with difficulty again, without any other change, may result in treatment continuation, possibly at high doses. How relevant this may be to the subject's quality of life could be debatable. This would be different from being able to walk independently again from having had to use a stick (similar one point improvement, of the leg score from three to two). In the same way, although more extensive, the I-RODS may pose similar issues, with a four-point improvement as a result of feeling less difficulty making a sandwich, washing up, moving a chair or catching a ball (if the need ever occurred for either!), having less impact in the eyes of many active patients, 
than recovering the ability, albeit with difficulty, to run, dance, climb stairs or carry and put down a heavy load. Therapeutic decisions made on the basis of such similar point improvements, without consideration of their weight in terms of quality of life amelioration in the individual, is problematic. Lack of clear indication, in the first place, of the amplitude of change to be considered in absolute terms, may make this task undoable. A cut-off of $8 \mathrm{kPa}$ has been suggested as clinically meaningful with Martin vigorimeter grip strength measurements [8]. However its application in practice remains uncertain and debatable. Still more uncertain are the meaningful amplitude of improvements of timed $10-\mathrm{m}$ walks or 6-min walks with relevance to individual patients' deficits and functional needs to improve their quality of life in CIDP, although cut-offs have been proposed for other disorders [13].

Despite their limitations, available disability scales offer the best possible method for objective assessment of patients with CIDP. Given the high cost of IVIg and plasma exchanges and the side-effects that may occur with any treatment, it is imperative that evaluation of treatment benefit is based on as reliable and reproducible methods as possible. Disability scales appear to clearly offer significant advantages to make this process more consistent and achievable in practice. Besides issues directly related to the scales themselves, many challenges however remain, including the problems of concurrent illnesses, to be separated from purely psychological factors, which undeniably also play a role in the use of any scale. It appears likely that the available scales, although very useful, may frequently be insufficient and that their use should be part of a wider more detailed assessment, rather than represent it in totality. Instead of simpler, shorter and exclusively patient-reported outcomes as suggested by recent research and which may be appropriate and useful for future trials, the reality of clinical day-to-day practice may in fact, require more detailed and lengthier evaluations. These should ideally be tailored to each subject, given the complexity of each individual case and, especially, the importance of making the best management decision for each patient.

Financial \& competing interests disclosure

YA Rajabally has received speaker/consultancy honoraria from CSL Behring, LfB, Grifols, BPL, Octapharma and Kedrion, has received educational sponsorships from LfB, CSL Behring and Baxter and has obtained research grants from CSL Behring and LfB. The author has no other relevant affiliations or financial involvement with any organization or entity with a financial interest in or financial conflict with the subject matter or materials discussed in the manuscript apart from those disclosed.

No writing assistance was utilized in the production of this manuscript.

\section{References}

1. Rajabally YA, Simpson BS, Beri S, Bankart J, Gosalakkal JA. Epidemiologic variability of chronic inflammatory demyelinating polyneuropathy with different diagnostic criteria: study of a UK population. Muscle Nerve 39(4), 432-438 (2009).

2. Eftimov F, Winer JB, Vermeulen M, de Haan R, van Schaik IN. Intravenous immunoglobulin for chronic inflammatory demyelinating polyradiculoneuropathy. Cochrane Database Syst Rev. 12, Cd001797 (2013).

3. Vanhoutte EK, Faber CG, van Nes SI $e$ t al. Modifying the Medical Research Council grading system through Rasch analyses. Brain 135(Pt 5), 1639-1649 (2012).

4. Hughes R, Bensa S, Willison $\mathrm{H}$ et al. Randomized controlled trial of intravenous immunoglobulin versus oral prednisolone in chronic inflammatory demyelinating polyradiculoneuropathy. Ann. Neurol. 50(2), 195-201 (2001).

5. Graham RC, Hughes RA. A modified peripheral neuropathy scale: the overall neuropathy limitations scale. J. Neurol. Neurosurg. Psych. 77(8), 973-976 (2006).

6. Breiner A, Barnett C, Bril V. INCAT disability score: a critical analysis of its measurement properties. Muscle Nerve 50(2), 164-169 (2014).

7. van Nes SI, Vanhoutte EK, van Doorn PA et al. Rasch-built overall disability scale (R-ODS) for immune-mediated peripheral neuropathies. Neurology 76(4), 337-345 (2011).

8. Vanhoutte EK, Latov N, Deng C et al. Vigorimeter grip strength in CIDP: a responsive tool that rapidly measures the effect of IVIG: the ICE study. Eur. J. Neurol. 20(5), 748-755 (2013).

9. Draak TH, Gorson KC, Vanhoutte EK et al. Correlation of the patient's reported outcome Inflammatory-RODS with an objective metric in immune-mediated neuropathies. Eur. J. Neurol. 23(7), 1248-1253 (2016).

10. Rajabally YA, Narasimhan M. Jamar hand-held grip dynamometry in chronic inflammatory demyelinating polyneuropathy. J. Neurolog. Sci. 325(1-2), 36-38 (2013).

11. Vanhoutte EK, Draak TH, Gorson KC et al. Impairment measures versus inflammatory RODS in GBS and CIDP: a responsiveness comparison. JPNS 20(3), 289-295 (2015).

12. Alabdali M, Abraham A, Alsulaiman A et al. Clinical characteristics and impairment and disability scale scores for different CIDP disease activity status classes. J. Neurolog. Sci. 372, 223-227 (2017). 
13. Hobart J, Blight AR, Goodman A, Lynn F, Putzki N. Timed 25-foot walk: direct evidence that improving $20 \%$ or greater is clinically meaningful in MS. Neurology 80(16), 1509-1517 (2013). 